\title{
Phylogenetic analysis of spotted fever group rickettsiae by study of the outer surface protein rOmpA
}

\author{
P.-E. Fournier, V. Roux and D. Raoult
}

Author for correspondence: D. Raoult. Tel: +33491324375. Fax: +33491387772.

e-mail: Didier.Raoult@medecine.univ-mrs.fr

Unité des rickettsies, CNRS UPRES-A 6020, Faculté de Médecine, Université de la Méditerranée, 27 Boulevard Jean Moulin, 13385 Marseille cedex 05, France

\begin{abstract}
Rickettsiae are classified in the order Rickettsiales and have been included in the $\alpha$ subclass of the class Proteobacteria on the basis of 16S rRNA gene sequence comparison. To estimate the evolutionary forces that have shaped the members of the spotted fever group (SFG) rickettsiae, the ompA gene (apart from the tandem repeat units), encoding an antigenic high-molecularmass membrane protein specific for the group, was amplified and sequenced from 21 isolates. The phylogenetic relationships between SFG rickettsiae were inferred from the comparison of both the gene and derived protein sequences, using the parsimony, neighbour-joining and maximum-likelihood methods. Three strongly supported phylogenetic sub-groups were distinguished: first, the Rickettsia conorii complex (R. conorii Malish, R. conorii M1, R. conorii Moroccan, $R$. conorii Indian tick typhus, Astrakhan fever rickettsia and Israeli tick typhus rickettsia); second, a cluster including Rickettsia africae, strain $S$, Rickettsia parkeri, Rickettsia sibirica and 'Rickettsia mongolotimonae '; and, third, a cluster including Rickettsia aeschlimannii, Rickettsia rhipicephali, Rickettsia massiliae, Bar 29 and Rickettsia montanensis. Rickettsia rickettsii, Rickettsia japonica, Rickettsia slovaca and Thai tick typhus rickettsia did not cluster with any other Rickettsia species. To test whether positive selection was responsible for sequences diversity, rates of synonymous and nonsynonymous nucleotide substitutions were compared for Rickettsia ompA alleles and indicated that this gene is undergoing neutral evolution.
\end{abstract}

Keywords: spotted fever group rickettsiae, phylogenetic analysis, outer-membrane protein rOmpA, Rickettsia

\section{INTRODUCTION}

The Rickettsia species are small, highly fastidious Gram-negative bacteria that form a monophyletic cluster within the $\alpha$ subclass of the class Proteobacteria. Traditionally, Rickettsia species have been divided into two groups, namely the typhus group (TG) and spotted fever group (SFG), on the basis of the clinical presentation, immunological reactivity, DNA G $+\mathrm{C}$ $\mathrm{mol} \%$ content and intracellular position (Weiss \& Moulder, 1984). All species are obligatorily intracellular and maintain a life cycle in mammal cells or arthropods. In the SFG, rickettsiae are maintained by

Abbreviations: MIF, microimmunofluorescence test; SFG, spotted fever group; SPD, specificity difference; TG, typhus group.

The GenBank accession numbers for the sequences in this paper are listed in Methods. both transovarial transmission and horizontal acquisition of organisms by means of ingestion of infested blood meals. Some SFG rickettsiae are human pathogens, causing tick-borne eruptive fevers, whereas others have only been isolated from arthropods (Raoult \& Roux, 1997). Endothelial cells are specifically infected by rickettsiae, which are thus responsible for various disseminated infections. SFG rickettsiae have been isolated worldwide, but their geographical distribution varies depending of the rickettsia. The number of the SFG representatives has increased following the development of improved cell culture isolation techniques (Marrero \& Raoult, 1989) and an increased interest in arthropod-transmitted diseases. Several studies aimed at assessing the genotypic relationships of these rickettsiae have been performed using DNA sequence comparison. Stothard \& Fuerst (1995) and Roux \& Raoult (1995) inferred 
phylogenies based on the comparison of aligned $16 \mathrm{~S}$ rRNA gene sequences.

Subsequently, Roux et al. (1996a) inferred phylogenetic relationships between Rickettsia species from the comparison of the faster-evolving citrate-synthaseencoding gene ( $g l t A)$ sequences, showing that this tool is more sensitive than $16 \mathrm{~S}$ rRNA comparison, but reliable phylogenetic inter-relationships could only be drawn among rickettsiae that diverged early from the common ancestor of SFG. The rOmpA-encoding gene was considered a good candidate for a phylogenetic analysis of most of the SFG representatives because it has been shown to exhibit higher degrees of interspecies difference than both the 16S rRNA gene and glt $A$ by PCR-RFLP and sequencing (Eremeeva et al., 1994b; Regnery et al., 1991). Furthermore, as immune serum to rOmpA reacts with all the representatives of the SFG and Rickettsia canadensis, it appeared that the protein, and therefore the gene, were present among all SFG members (Anacker et al., 1987; Vishwanath, 1991). A cloned portion of the Rickettsia rickettsii and Rickettsia conorii omp $A$ gene has been shown to induce the expression of a truncated recombinant protein that elicits a protective immune response in mice and guinea pigs against a normally lethal inoculum of $R$. rickettsii and $R$. conorii (Sumner et al., 1995; Vishwanath et al., 1990). Moreover, rOmpA and rOmpB, another high-molecular-mass surface protein, are the antigenic determinants of serotyping in mice, which is the currently preferred method for identification of SFG rickettsiae species (Philip et al., 1978).

During their evolution, rickettsiae may have been exposed to selective forces imposed by factors affecting their arthropod or mammalian hosts. Among those species that infect mammals, one could therefore expect this selective pressure to be directed against their immunoexposed proteins. Such a selection exerted by the human immune system has been demonstrated to occur against immunogenic surface proteins of other pathogenic organisms, including Plasmodium falciparum (Hughes, 1991) and Neisseria gonorrhoeae (Smith et al., 1995). Thus, rOmpA could be a good candidate for estimating the effects of positive selection: the protein has been characterized only in SFG rickettsiae, is surface exposed (Walker et al., 1992) and elicits a strong immune reaction during infection.

The omp $A$ gene has previously been completely sequenced in two species of Rickettsia, $R$. rickettsii and $R$. conorii (Anderson et al., 1990; Crocquet-Valdes et $a l ., 1994)$. In this work, we report the DNA sequence of the entire $o m p A$ gene, excluding the central region containing tandem repeat units, of 21 SFG rickettsiae isolates. From the alignment of these sequences, we inferred the phylogenetic relationships within the SFG. Furthermore, to examine whether the evolution of the protein could be related to a positive selection, we have analysed the substitution rates between pairs of sequences.

\section{METHODS}

Rickettsial strains. The strains used in this study are listed in Table 1.

Rickettsial cultivation and nucleic acid preparation. The rickettsiae were propagated on Vero cell (ATCC CRL-1587) monolayers in Eagle's minimal essential medium (MEM; Seromed) supplemented with $4 \%$ fetal calf serum (Seromed) and $2 \mathrm{mM}$ glutamine, at $32{ }^{\circ} \mathrm{C}$. Harvesting of the cells was done when the degree of infection as estimated by Gimenez staining was high (3-5 d). Rickettsial cultures were centrifuged ( $12000 \mathrm{~g}$ for $10 \mathrm{~min})$, resuspended in MEM and stored at $-70^{\circ} \mathrm{C}$ until required.

Cells were lysed by overnight incubation in a mixture of $1 \%$ SDS and $0.2 \mathrm{mg}$ proteinase $\mathrm{K} \mathrm{ml}^{-1}$, in an Eppendorf tube at $37^{\circ} \mathrm{C}$. A phenol extraction and then a phenol/chloroform extraction were carried out after recovery of the aqueous phase, and DNA was precipitated by addition of two volumes of chilled ethanol held overnight at $-20^{\circ} \mathrm{C}$. DNA was pelleted by centrifugation $(12000 \mathrm{~g}$ for $10 \mathrm{~min})$, then washed with cold $70 \%$ ethanol. After centrifugation, the pellet was then dried and resuspended in sterile distilled water.

PCR amplification and sequencing method. The primer $\mathrm{Rr}$ 190-70p has been described by Regnery et al. (1991). Other potentially useful primers were designed following identification of suitable hybridization sites with conserved regions of the gene after alignment of the sequences of $R$. rickettsii (Anderson et al., 1990) and $R$. conorii (strain Malish) (Croquet-Valdes et al., 1994). These primers are described in Table 2. For PCR amplification, one primer was labelled at the $5^{\prime}$ end with biotin during oligonucleotide synthesis (Eurogentec) whereas the other was unmodified. Two microlitres of the DNA preparation was amplified in a $100 \mu \mathrm{l}$ reaction mixture containing $10 \mathrm{pmol}$ each primer; $200 \mu \mathrm{M}$ (each) dATP, dCTP, dGTP and dTTP (Boerhinger Mannheim); 1.25 U AmpliTaq DNA polymerase (PerkinElmer Cetus); $6 \mu \mathrm{l}$ of a $25 \mathrm{mM}$ solution of $\mathrm{MgCl}_{2}$ (PerkinElmer) in $1 \times T a q$ buffer. Amplifications were carried in a DNA thermal cycler (PTC 200; MJ Reasearch) under the following conditions: an initial $3 \mathrm{~min}$ of denaturation at $95^{\circ} \mathrm{C}$ was followed by 35 cycles of denaturation for $20 \mathrm{~s}$ at $95^{\circ} \mathrm{C}$, annealing for $30 \mathrm{~s}$ at $46^{\circ} \mathrm{C}$ and extension for $1 \mathrm{~min}$ at $65^{\circ} \mathrm{C}$. The amplification was completed by holding for 7 min at $72^{\circ} \mathrm{C}$ to allow complete extension of the PCR products. Purification of PCR products and sequencing reactions were performed as previously described (Roux et al., 1996a). Sequencing reactions were resolved on $6 \%$ polyacrylamide gels (Ready Mix Gels, ALF grade; Pharmacia Biotech) and electrophoresis was performed in the ALF DNA sequencer (Pharmacia Biotech) in $1 \times \mathrm{TBE}$ buffer, pH 8 (44.5 mM Tris $/ \mathrm{HCl}, 44.5 \mathrm{mM}$ boric acid, $1 \mathrm{mM}$ EDTA), according to the manufacturer's instructions.

\section{Data analysis}

(i) Determination of phylogenetic relationships. The ompA sequences were translated into protein sequences using PC/GENE software. The $o m p A$ sequences and rOmpA amino acid sequences were aligned using the multisequence alignment program CLUSTAL within the BISANCE environment (Dessen et al., 1990). The percentages of similarity were determined using the DNASIS software package (Hitachi Software Engineering). The phylogenetic relationships between the studied representatives of the genus Rickettsia were determined using version 34 of the PHYLIP software (Felsenstein, 1989). Distance matrices generated by DNADIST 


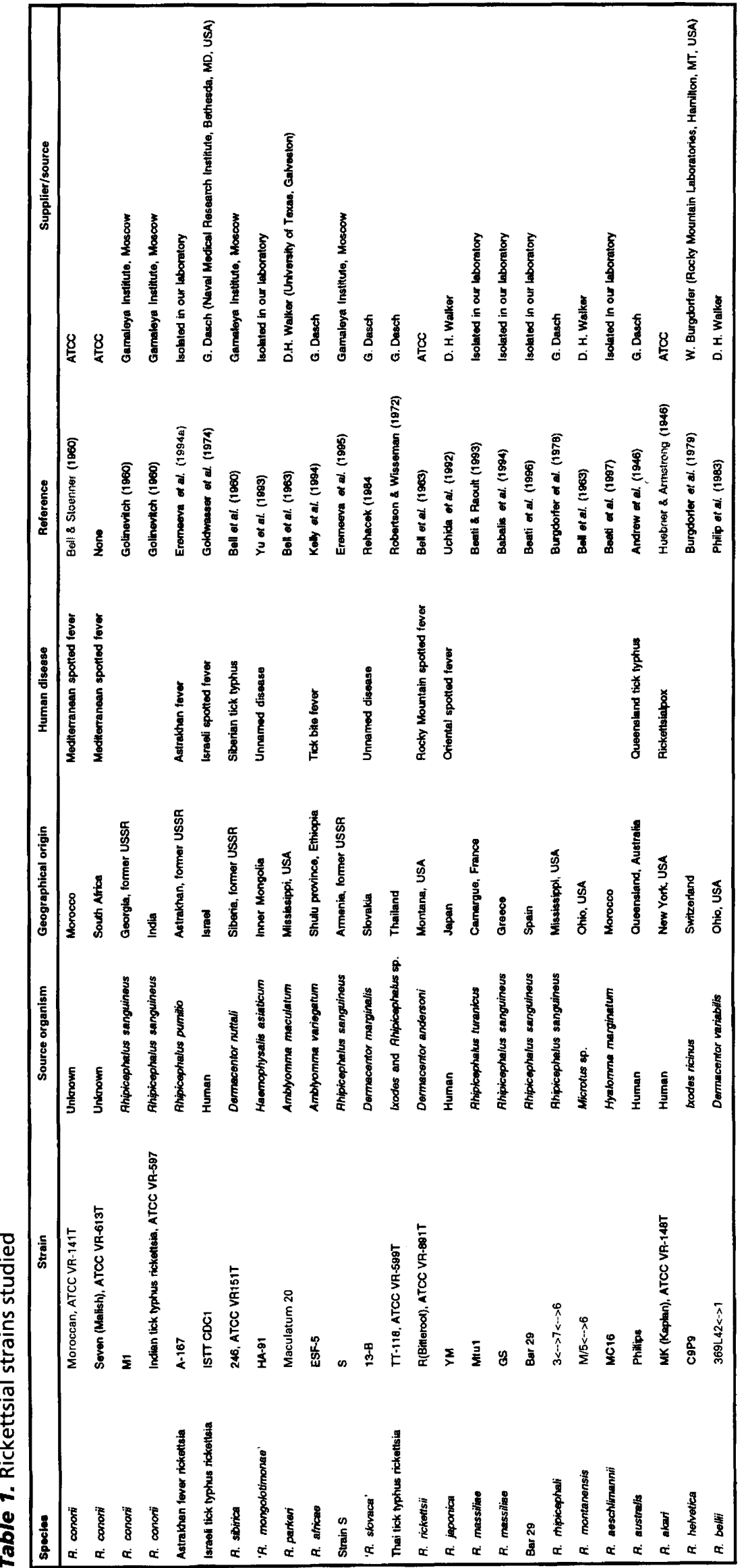


Table 2. Oligonucleotide primers

\begin{tabular}{|c|c|c|c|}
\hline Fragment & Primer & Nucleotide sequence & $\begin{array}{c}\text { ompA } \\
\text { position* }\end{array}$ \\
\hline \multirow[t]{4}{*}{ I } & $190-70 \dagger \ddagger$ & ATGGCGAATATTTCTCCAAAA & $70-90$ \\
\hline & $190-264 \ddagger$ & ATGGCGAATATTTCTCCAAAA & 264-245 \\
\hline & $190-485 \ddagger$ & GCAAAAGCTTAACTTTAAA & $485-503$ \\
\hline & $190-701 \dagger \ddagger$ & GTTCCGTTAATGGCAGCATCT & $701-681$ \\
\hline \multirow[t]{5}{*}{ II } & $190-3588 \dagger \ddagger$ & AACAGTGAATGTAGGAGCAG & $3588-3607$ \\
\hline & $190-3817 \ddagger$ & CTGCAACTGTTCCTATAG & $3817-3800$ \\
\hline & $190-3968 \ddagger$ & TAGCAGCTGATTTAGTAGCT & $3968-3986$ \\
\hline & $190-4084 \ddagger$ & CATCACCGATATTTCTAGC & $4084-4065$ \\
\hline & $190-4406+\ddagger$ & ACTATACCCTCATCGTCATT & $4406-4387$ \\
\hline \multirow[t]{5}{*}{ III } & $190-4338+t$ & TTCAGGAAACGACCGTACG & $4338-4356$ \\
\hline & $190-4710 \ddagger$ & AACATTTACATTGTTATTTA & $4710-4690$ \\
\hline & $190-4859$ & GCGAAATCCAAGGTACAGG & $4859-4877$ \\
\hline & $190-5044 \ddagger$ & AACTTGTAGCACCTGCCGT & $5044-5028$ \\
\hline & $190-5238+\ddagger$ & ACTATTAAAGGCTAGGCTATT & $5238-5218$ \\
\hline \multirow[t]{6}{*}{ IV } & $190-5125+t$ & GCGGTTACTTTAGCCAAAGG & $5125-5144$ \\
\hline & $190-5498 \ddagger$ & CAGCAGGTGGTTTAAAGCCT & $5498-5517$ \\
\hline & $190-5561 \ddagger$ & CGGTTGTCATTATTAATAG & $5561-5543$ \\
\hline & $190-5768 \ddagger$ & CACCGCTACAGGAAGCAGAT & $5768-5787$ \\
\hline & $190-5831 \ddagger$ & GTGTCGCTAGGTTTTACAAC & $5831-5812$ \\
\hline & $190-6013 \dagger \ddagger$ & TCTTCTGCGTTGCATTACCG & 6013-5994 \\
\hline \multirow[t]{6}{*}{$\mathrm{V}$} & $190-5917 \dagger t$ & TCAGGGAATAAAGGTCCTG & $5917-5935$ \\
\hline & $190-6092 \ddagger$ & AGATCATCGCTAACGAAGCC & $6092-6073$ \\
\hline & $190-6228 \ddagger$ & CGTTGAAGCTATAGCATC & $6228-6245$ \\
\hline & $190-6427 \ddagger$ & ATCTAAGCCCAGCTAGCGGT & $6427-6408$ \\
\hline & $190-6585 \ddagger$ & CGCAATGGTCGATTATGC & $6585-6602$ \\
\hline & $190-6808 \dagger \ddagger$ & CACGAACTTTCACACTACC & $6808-6790$ \\
\hline
\end{tabular}

* Numbering refers to the rOmpA sequence of $R$. rickettsii reported by Regnery et al. (1991).

$\dagger$ Oligonucleotide primer used for PCR amplification.

$\ddagger 5^{\prime}$-Isothiocyanate-labelled primer used for sequencing.

were determined under the assumptions of Jukes \& Cantor (1969) and Kimura (1980). These matrices were used to elaborate dendrograms using the neighbour-joining method (Saitou \& Nei, 1987). Two other dendrograms were constructed using data processing with the maximumlikelihood and the parsimony program DNAPARS. Bootstrap replicates were performed to estimate the node reliability of the phylogenetic trees obtained by the parsimony, maximum-likelihood and neighbour-joining methods (Brown, 1994). The bootstrap values were obtained from 100 trees generated randomly with SEQBOOT and CONSENSE in the PHYLIP software package.

The phylogenetic relationships inferred from protein were determined using the neighbour-joining, parsimony and maximum-likelihood methods with PROTDIST, PROTPARS and DNAML, respectively, in the PHYLIP software.

(ii) Calculation of synonymous and non-synonymous substitution rates per site. These values were determined from the entire sequence of ompA by the method of Nei \& Gojobori (1986) within the MEGA suite (Kumar et al., 1993). The significance of the $d_{\mathrm{s}} / d_{\mathrm{N}}$ value was estimated using Student's $t$-test. An equal frequency of synonymous and non-synonymous substitutions (with a $d_{\mathrm{s}} / d_{\mathrm{N}}$ ratio close to 1 ) indicates that the entire gene is undergoing neutral evolution. However, a ratio of $>1$ indicates that purifying selection is occurring, whereas a value $<1$ suggests that there is positive selection for amino acid sequence variation.

Nucleotide sequence accession numbers. The GenBank accession numbers for the nucleotide sequence data reported in this paper are as follows: U83442 for Rickettsia japonica, U83445 for Rickettsia massiliae strain Mtu1, U83444 for $R$. massiliae strain GS, U83447 for Rickettsia montanensis, U83450 for Rickettsia rhipicephali, U83438 for Bar 29, U83446 for Rickettsia aeschlimannii, U83451 for $R$. rickettsii, U83454 for 'Rickettsia slovaca', U83456 for Thai tick typhus rickettsia, U83455 for Rickettsia sibirica, U83439 for 'Rickettsia mongolotimonae', U83449 for Rickettsia parkeri, U83436 for Rickettsia africae, U83452 for strain S, U83441 for Israeli tick typhus rickettsia, U83437 for Astrakhan fever rickettsia, U83448 for $R$. conorii strain Moroccan, U83440 for $R$. conorii strain Indian tick typhus, $\mathrm{U} 83453$ for $R$. conorii strain Malish and U83443 for $R$. conorii strain M1. The GenBank accession numbers for the first part of the ompA sequences included in Fig. 1 are as follows: $\mathrm{U} 43795$ for $R$. japonica, U43799 for $R$. massiliae strain Mtu1, U43793 for R. massiliae strain GS, U43801 for $R$. montanensis, $\mathrm{U} 43803$ for $R$. rhipicephali, $\mathrm{U} 43792$ for Bar 29 , U43800 for $R$. aeschlimannii, U43804 for $R$. rickettsii, 
U43808 for ' $R$. slovaca', U43809 for Thai tick typhus rickettsia, U43807 for $R$. sibirica, $\mathrm{U} 43796$ for ' $R$. mongolotimonae', U43802 for $R$. parkeri, U43790 for $R$. africae, U43805 for strain S, U43797 for Israeli tick typhus rickettsia, U43791 for Astrakhan fever rickettsia, U45244 for $R$. conorii strain Moroccan, U43794 for $R$. conorii strain Indian tick typhus, U43806 for $R$. conorii strain Malish and $\mathrm{U} 43798$ for $R$. conorii strain M1.

\section{RESULTS}

\section{PCR amplification}

ompA fragments were amplified from all the studied strains of the SFG rickettsiae except $R$. australis, $R$. helvetica, R. akari and R. bellii, for which none of the primer pairs used could amplify any fragment. For each strain, the omp $A$ gene, excluding the central tandem repeat region, was amplified in five fragments. The 5' end fragment of the gene was amplified using $\mathrm{Rr}$ $190.70 \mathrm{p}$ (Regnery et al., 1991) and a consensus primer 190-701 that hybridized to the ompA gene close to the $5^{\prime}$ end of the tandem repeat units (Roux et al., 1996b). The size of PCR product was 632 bp for all strains except $R$. conorii strain M1 (476 bp), $R$. conorii strain Moroccan (608 bp), $R$. montanensis $(629 \mathrm{bp})$ and $R$. conorii strain Indian tick typhus rickettsia (635 bp). The other four overlapping fragments were amplified using four consensus primer pairs. Primer 190-3588 hybridized to the $o m p A$ gene close to the $3^{\prime}$ end of the tandem repeat units. The second amplified fragment was 819 bp long for all strains except Thai tick typhus rickettsia (813 bp), ' $R$. mongolotimonae' (804 bp), $R$. sibirica (813 bp), Astrakhan fever rickettsia (822 bp) and Israeli tick typhus rickettsia ( $822 \mathrm{bp}$ ). The third fragment was $901 \mathrm{bp}$ long for all strains except $R$. massiliae strains Mtul (904 bp) and GS (904 bp), Bar29 (904 bp), $R$. rhipicephali (904 bp) and $R$. aeschlimannii (904 bp). The fourth fragment was $889 \mathrm{bp}$ long for all strains except $R$. massiliae strains Mtul (842 bp) and GS (842 bp), Bar29 (842 bp) and $R$. rhipicephali $(842 \mathrm{bp}$ ). The fifth fragment was $892 \mathrm{bp}$ long for all strains. The insertions and/or deletions which yielded these size variations are presented in Table 3.

\section{Sequencing}

The sequence of both DNA strands was determined twice. The same sequence was obtained for two isolates of the species $R$. massiliae, GS and Mtu1. Unique

Table 3. Positions of the deletions and insertions characterized in the SFG rickettsiae studied. The positions are numbered with regard to the sequence of $R$. rickettsii

Numbering refers to positions in the nucleotide sequence of the five $o m p A$ fragments joined end-to-end and renumbered from 1 to 3785. Fragment $I$ is derived from the sequenced insert between positions 70 and 701 and is renumbered from 1 to 591 . Fragment II is derived from the sequenced insert between positions 3588 and 4406 and is renumbered from 592 to 1375 . Fragment III is derived from the sequenced insert between positions 4338 and 5238 and is renumbered from 1347 to 2210 . Fragment IV is derived from the sequenced insert between positions 5125 and 6013 and is renumbered from 2137 to 2990 . Fragment V is derived from the sequenced insert between positions 5917 and 6808 and is renumbered from 2934 to 3785 . ${ }^{\mathrm{d}}$, Deletion; ${ }^{\mathrm{i}}$, insertion.

\begin{tabular}{|c|c|c|c|c|c|}
\hline Rickettsia & Fragment I & Fragment II & Fragment III & Fragment IV & Fragment V \\
\hline$R$. conorii strain Malish & $\begin{array}{l}\text { TTT }^{\mathrm{i}}: 101-103 \\
\text { AAT }^{\mathrm{d}}: 160-162\end{array}$ & & & & \\
\hline R. conorii strain $\mathrm{M} 1$ & $\begin{array}{l}\text { TTT }^{i}: 101-103 \\
\text { AAT }^{\mathrm{d}}: 160-162 \\
156 \text { bases }^{\mathrm{d}}: 238-393\end{array}$ & & & & \\
\hline R. conorii strain Moroccan & $\begin{array}{l}\text { TTT }^{\mathrm{i}}: 101-103 \\
\text { AAT }^{\mathrm{d}}: 160-162 \\
24 \text { bases }^{\mathrm{d}}: 360-383\end{array}$ & & & & \\
\hline R. conorii strain Indian & TTT $^{\mathrm{i}}: 101-103$ & & & & \\
\hline Astrakhan fever rickettsia & & $\mathrm{AAT}^{\mathrm{i}}: 872-874$ & & & \\
\hline Israeli tick typhus rickettsia & & $\mathrm{AAT}^{i}: 872-874$ & & & \\
\hline Thai tick typhus rickettsia & & $\begin{array}{l}\text { TGA }^{\mathrm{d}}: \text { 679-681 } \\
\text { CTT }^{\mathrm{d}}: 767-769\end{array}$ & & & \\
\hline R. aeschlimannii & & & $\mathrm{GGT}^{\mathrm{i}}: 1631-1633$ & & \\
\hline$R$. montanensis & $\mathrm{CTA}^{\mathrm{d}}: 119-121$ & $\mathrm{CAC}^{\mathrm{d}}: 1350-1352$ & & & \\
\hline Bar 29 & & & GGT $^{i}: 1631-1633$ & TGC $^{i}: 2288-2290$ & \\
\hline R. massiliae & & & $\mathrm{GGT}^{\mathrm{i}}: 1631-1633$ & TGC $^{i}: 2288-2290$ & \\
\hline R. rhipicephali & & & $\mathrm{GGT}^{\mathrm{i}}: 1631-1633$ & $\mathrm{TGC}^{\mathrm{i}}: 2288-2290$ & \\
\hline R. sibirica & & ACGCAA $^{d}:$ 693-698 & & & \\
\hline ' $R$. mongolotimonae' & & $\begin{array}{l}\text { CCTCTTGAT }^{\mathrm{d}}: 674-682 \\
\text { ACGCAA }^{\mathrm{d}}: 693-698\end{array}$ & & & \\
\hline
\end{tabular}




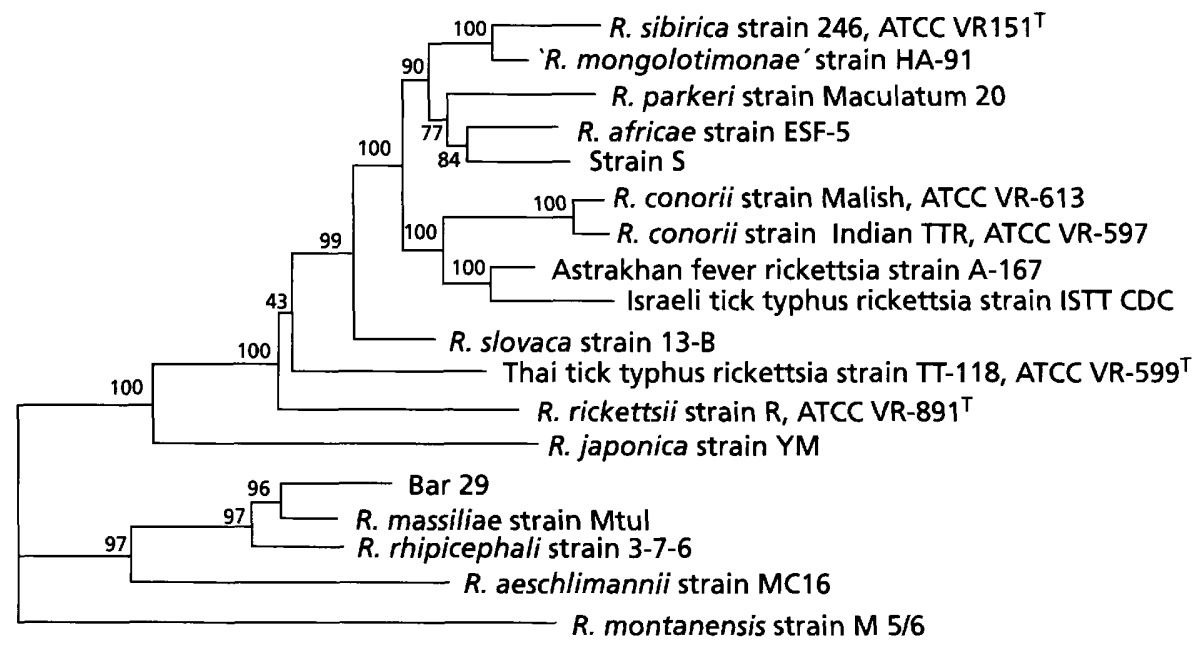

$1.5 \%$

Fig. 1. Phylogenetic tree of bacteria belonging to the SFG rickettsiae inferred from comparison of the ompA gene sequences. The sequences were aligned using the multisequence alignment program CLUSTAL, which is a part of the BISANCE Software package. Phylogenetic relationships were inferred with version 3.4 of the PHYLIP software package. The evolutionary distance values were determined by the method of Kimura (1980), and these values were used to construct a dendrogram by the neighbour-joining method. The scale bar represents $1.5 \%$ difference in nucleotide sequences. The numbers at nodes are the proportion of 100 bootstrap resamplings that support the topology shown.

sequences were found for each of four strains of the species $R$. conorii. The sequenced fragments of the omp $A$ gene were analysed from base position 91 to 680 and from 3608 to 6789 with respect to the $R$. rickettsii published sequence (Anderson et al., 1990). The first and second fragments were united and the complete sequence was renumbered from base 1 to base 3785 .

\section{Sequence comparison}

(i) Nucleotide sequences. The nucleotide sequences obtained for $R$. rickettsii and $R$. conorii strain Malish were identical to those reported previously (Anderson et al., 1990; Crocquet-Valdes et al., 1994). Variations in the sizes of both the $5^{\prime}$-end and the $3^{\prime}$-end fragments of ompA were observed among the SFG strains. However, the most important source of genetic variation between rickettsiae was due to base substitutions, which are scattered throughout the entire sequence. Of the 489 sites at which point mutations occurred within the rOmpA-encoding gene, $173(35.4 \%)$ occurred in the first position of a codon, $164(33.5 \%)$ in the second position and $152(31.1 \%)$ in the third position. Among mutations, $345(70.5 \%)$ were transitions and 144 $(29.5 \%)$ were transversions. Sequence similarities ranged from $95.2 \%$ ( $R$. montanensis $/ R$. conorii Malish) to $100 \%$ (R. massiliae GS/R. massiliae Mtu1).

(ii) Amino acid sequences. Of the 489 sites at which a base substitution was found, $148(30 \cdot 3 \%)$ produced nonsynonymous amino acid changes. Sequence similarities ranged from $89 \%$ between $R$. montanensis and $R$. conorii strain Moroccan to $100 \%$ between the two $R$. massiliae strains.

\section{Phylogeny inference}

The dendrograms obtained with the three different tree-building analysis methods used showed similar organization for most of the rickettsiae whether inferred from the gene or the derived protein sequences. Only the positions of $R$. rickettsii and Thai tick typhus rickettsia were unstable, as is reflected in low bootstrap value at the node from which branched $R$. rickettsii $(43 \%)$. All other nodes were wellsupported ( $>70 \%$ bootstrap values) (Fig. 1). We identified three monophyletic groups within the tree: one made of $R$. aeschlimannii, R.rhipicephali, $R$. massiliae, $R$. montanensis and Bar 29, another including the $R$. conorii complex ( $R$. conorii strains, Astrakhan fever rickettsia and Israeli tick typhus rickettsia) and a third including $R$. africae, strain $\mathrm{S}$, $R$. parkeri, $R$. sibirica and ' $R$. mongolotimonae'. $R$. rickettsii, $R$. slovaca, Thai tick typhus rickettsia and $R$. japonica did not cluster with any other Rickettsia species.

\section{$d_{\mathrm{s}}$ and $d_{\mathrm{N}}$ values}

Mean percentages of synonymous and non-synonymous substitutions per site were determined from the entire sequence. The $d_{\mathrm{S}}$ and $d_{\mathrm{N}}$ values were $2 \cdot 85 \pm 0.6$ was $2 \cdot 87 \pm 0 \cdot 3$, respectively. These values are significantly different $\left(P<10^{-6}\right)$. A mean overall $d_{\mathrm{S}} / d_{\mathrm{N}}$ value 
of 0.99 suggests that this gene is undergoing neutral evolution.

\section{DISCUSSION}

Phenotypic criteria (morphology and antigenic and metabolic characters) have long been the basis of bacterial taxonomy. However, since the 1960s, molecular approaches to the study of evolution have involved sequence analysis of proteins (cytochrome $c$, fibrinopeptides, ferrodoxins ...) (Wilson et al., 1977). In the 1980s, PCR and rapid sequencing techniques for DNA became available and comparison gene sequences became the most useful tool to make phylogenetic inferences. It was established that the most adapted molecular chronometers were universal molecules with highly conserved function that were established at early stages in evolution, functions that are not affected by changes in the organism's environment. Genes encoding rRNA were chosen universally as molecular chronometers (Woese, 1987). The bacterial phylogeny that emerged from molecular sequence comparisons had little in common with preconceptions. This is particulary true in the order Rickettsiales, a taxon that was originally a repository for intracellular bacteria, which, by their nature, could be characterized only on the basis of a few non-specific phenotypic criteria. Phylogeny-based taxonomic reconstructions have led to numerous genera being removed from the order [Rochalimaea (Weisburg et al., 1991), Coxiella (Weisburg et al., 1989), Rickettsiella (Roux et al., 1997), Eperythrozoon, Hemobartonella (Rikihisa et al., 1997)], renamed [Rochalimaea/ Bartonella (Brenner et al., 1993), Grahamella/ Bartonella (Birtles et al., 1995)] or created [Orientia (Tamura et al., 1995)], and it was found that the taxonomy inside the cluster including Ehrlichia, Wolbachia, Anaplasma, Cowdria and Neorickettsia species is inadequate. The evolutionary relationhips among the members of the genus Rickettsia were first assessed through phylogenetic studies based on analysis of sequences of gene encoding 16S rRNA (Roux \& Raoult, 1995; Stothard \& Fuerst, 1995). However, the close evolutionary proximity of species, reflected by very highly conserved $16 \mathrm{~S}$ rRNA gene sequences, prevented a stable, well-supported phylogeny from being obtained. The relative 'insensibility' of $16 \mathrm{~S}$ rRNA gene-based assessments for inferring intragenus phylogenetic relationships is not limited to Rickettsia species (Birtles \& Raoult, 1996; Hookey et al., 1993; Martinez-Murcia et al., 1992). After the period of emphasis placed on rRNA analysis, genes encoding universally distributed and conserved proteins are now receiving renewed attention in those instances where less conserved chronometers are needed and the reliability of phylogenetic pattern derived from nucleic acid analyses needs to be tested by markers with different evolutionary origins (Olsen \& Woese, 1993). In this way, Roux et al. (1996a) have completed a comparative sequence analysis of the citrate synthase gene among Rickettsia species. How- ever, no reliable phylogenetic analysis for all the SFG representatives could be inferred from this study as the sequences derived from different species were too similar. Not all molecular chronometers are equally useful for reconstructing phylogenetic analyses. Some are restricted to particular taxonomic levels and/or particular bacterial groups. This is true among members of the same genus, in which case an additional more rapidly evolving housekeeping molecule is required as an alternative phylogenetic tool. In this work, we obtained base sequence data for all but the tandem repeat region of the $o m p A$ gene from SFG rickettsiae, allowing us to infer a well-supported phylogenetic study for most of the SFG rickettsiae, thus extending the phylogenetic studies of the entire order Rickettsiales and the genus Rickettsia undertaken with the genes encoding the 16S rRNA (Roux \& Raoult, 1995; Stothard \& Fuerst, 1995) and citrate synthase (Roux et al., 1996a).

The usefulness of ompA gene comparison for the differentiation of SFG rickettsiae was first recognized by Regnery et al. (1991), who demonstrated a clear heterogeneity in the gene sequences of different SFG species using restriction endonuclease analysis of PCRamplified ompA gene fragments. It was clear from these studies that intra-SFG variability in $o m p A$ was markedly higher than variability in either the $16 \mathrm{~S}$ rRNA or the glt $A$ genes. By obtaining complete $o m p A$ sequences except for the repeat domain, we were able to confirm and quantify these earlier observations.

Within the SFG, we have identified three distinct phylogenetic groups. Inside the first one clustered Eurasian isolates, the four $R$. conorii strains (M1, Moroccan, Malish and Indian tick typhus), Astrakhan fever rickettsia and Israeli tick typhus rickettsia, forming the $R$. conorii complex. $R$. africae, strain $\mathrm{S}, R$. parkeri, ' $R$. mongolotimonae' and $R$. sibirica clustered together and formed the second group. At the present time, $R$. africae has been isolated only in Africa, $R$. parkeri in the USA and $R$. sibirica, ' $R$. mongolotimonae' and strain $\mathrm{S}$ in Eurasia. The third group included $R$. massiliae, Bar 29 and $R$. aeschlimannii, encountered around the Mediterranean Sea, and $R$. montanensis and $R$. rhipicephali isolated from ticks in USA, and in USA and Europe, respectively.

The existence of the sub-group including $R$. massiliae is supported by dendrograms inferred from the comparison of $16 \mathrm{~S}$ rDNA, glt $A$ and omp $A$ sequences, and high bootstrap values were calculated for all supporting nodes using glt $A$ and omp $A$ analysis. The pathogenic potential in humans of all these rickettsiae is unknown at the present time, although a possible pathogenic role of Bar 29 in humans was recently proposed (Beati et al., 1996; Bella et al., 1991). The close evolutionary relationship between $R$. massiliae and Bar 29 is reflected in genotypic similarities. The species were isolated from Rhipicephalus tick species in France and Greece, and in Spain, respectively. $R$. rhipicephali has been also isolated from Rhipicephalus 
ticks in the USA and characterized in ticks in France (Drancourt et al., 1992) and Portugal (Bacellar et al., 1995), making it the only SFG rickettsia associated with ticks present in both the New and the Old Worlds. The other two representatives of this sub-group, $R$. aeschlimannii and $R$. montanensis, were isolated not from Rhipicephalus species but from Hyalomma and Dermacentor species, respectively. Recently, a phylogenetic study of ticks based on the comparison of mitochondrial 16S rDNA sequences suggested that Hyalomma species share a common ancestor with the Rhipicephalinae (Black \& Piesman, 1994), suggesting that the divergence pattern of the tick hosts parallels the recent divergence of the Rickettsia species that they carry.

The $R$. conorii complex was well-supported by omp $A$ derived phylogenetic analysis. This was not the case when analysis was performed by comparison of glt $A$ and 16S rDNA sequences. Human diseases are associated with these rickettsiae and, clinically, the main difference is the frequency of the eschar $(0,23$ and $72 \%$ for Israeli tick typhus, Astrakhan fever and Mediterranean spotted fever, respectively). The taxonomic position of Astrakhan fever rickettsia and Israeli tick typhus rickettsia remains unclear. Using the current criteria for the definition of a Rickettsia species, the microimmunofluorescence test (MIF), they belong to the same species ( $R$. conorii), as specificity difference (SPD) values are $\leqslant 3$ (Eremeeva et al., 1994a). However, the strains can easily be differentiated using phenotypic methods (SDS-PAGE and immunoblotting) and genotypic methods (restriction profiles of chromosomal DNA obtained by pulsedfield gel electrophoresis, 16S rDNA, glt $A$ and $o m p A$ sequencing). From an ecological perspective, Astrakhan fever rickettsia, Israeli tick typhus rickettsia and $R$. conorii were all isolated from Rhipicephalus ticks in Eurasia, but the geographical location of $R$. conorii is not restricted to a specific area as it has been isolated all around the Mediterranean Sea, in India, and around the Black Sea, and was isolated in Africa, where it was also found in Haemophysalis ticks. Reliance solely on the MIF for species identity is no longer justified, and the introduction of genotypic, phylogenetic and ecological criteria is required. In this way, species can be defined by polyphasic means.

The monophyletic relationship among members of the $R$. sibirica sub-group is evidenced by the phylogenetic analysis inferred from 16S rDNA, glt $A$ and omp $A$ sequence comparison. The geographical areas of distribution of ' $R$. mongolotimonae' (China and France) and $R$. sibirica (China, former USSR and Pakistan) overlap. These two rickettsiae are genotypically very close. ' $R$. mongolotimonae' was isolated from Haemophysalis asiaticum and $R$. sibirica from Haemophysalis concinna and Dermacentor species; thus horinzontal transfer of rickettsiae may have occurred after the separation of Dermacentor and Haemophysalis clades. The geographical dispersion of the other three representatives of this cluster, which are evolutionnarily close, is great, as $R$. africae, strain $\mathrm{S}$ and $R$. parkeri were isolated from Africa, Armenia and USA, respectively. Furthermore, their vectors (Rhipicephalus sanguineus for strain $\mathrm{S}$ and Amblyomma species for $R$. africae and $R$. parkeri) are phylogenically distant (Black \& Piesman, 1994).

The phylogenetic position of $R$. japonica was the same whichever analysis system was considered. It was intermediate between the $R$. massiliae sub-group and the other rickettsiae described, which presented a common ancestor.

$R$. rickettsii, ' $R$. slovaca' and Thai tick typhus rickettsia did not cluster with any other rickettsiae but branched alone. It seems that these species lacked a reliable phylogenetic analysis among the SFG.

The study of synonymous and non-synonymous substitutions allowed us to assess the manner of rOmpA evolution. Protein evolution depends on both the probability that a substitution will be compatible with the biochemical function of the protein and the dispensability of the protein to the organism. The regions of $o m p A$ we sequenced were undergoing neutral evolution. For free-living bacteria, the host immune system plays a major role as a positive selection factor. The obligate intracellular nature of Rickettsia sets them apart from the free-living bacteria, and thus the importance of host immunity on selection is unclear. The nature of the relationship between rickettsiae and arthropods suggests that their evolution is likely to be influenced far more by arthropod factors than those of transient warm-blooded hosts because rickettsiae are mainly maintained by transovarial transmission between tick generations. However, horizontal transfer of rickettsiae between vectors certainly occurs, as proven by the multiplicity of vectors for $R$. conorii or $R$. rickettsii (Raoult \& Roux, 1997). Furthermore, owing to their strict intracellular position, the mechanisms commonly implied in genetic transfer in free-living bacteria, such as plasmid-mediated conjugation, phage infection and transduction, transformation by unassociated DNA or conjugative transposition of mobile genetic elements, may have a low impact on the genetic evolution of rickettsiae. However, within tick hosts, co-infections with other bacteria such as Ehrlichia, Coxiella or Borrelia species may occur (P. Parola, L. Beati, M. Cambon \& D. Raoult, unpublished results). It is not clear whether there is intimate contact between different co-infectors, but, if so, genetic transfer may be possible. However, evidence for this is yet to be found.

Recently, new Rickettsia species were characterized in insects [AB bacterium (Werren et al., 1994) and pea aphid rickettsia (Chen et al., 1996)], attesting to the association of these bacteria with a large clade of arthropod hosts. In future, the goal should be to obtain more isolates from different hosts and from different geographical regions. Analysis including an enlarged panel of strains should reinforce and clarify our understanding of the phylogeny of rickettsiae. 
Moreover, to confirm our results and to obtain a precise and reliable phylogenetic position for all the SFG members, other bases for phylogenetic inference are required. Only very few genes have been sequenced for the SFG rickettsiae. The rOmpB protein gene, which is also strongly immunogenic and is common to the SFG and TG (Gilmore et al., 1991), may prove to be useful for this goal. In fact, it seems likely that outstanding phylogenetic analysis of micro-organisms will follow from comparison of part or whole bacterial chromosomes in their organization and in their sequencing.

\section{ACKNOWLEDGEMENTS}

The authors are very grateful to Bernadette Thiers for her technical assistance and to Richard J. Birtles for reviewing the manuscript.

\section{REFERENCES}

Anacker, R. L., McDonald, G. A., List, R. H. \& Mann, R. E. (1987). Neutralizing activity of monoclonal antibodies to heat-sensitive and heat-resistant epitopes of Rickettsia rickettsii surface proteins. Infect Immun 55, 825-827.

Anderson, B. E., McDonald, G. A., Jones, D. C. \& Regnery, R. L. (1990). A protective protein antigen of Rickettsia rickettsii has tandemly repeated, near-identical sequences. Infect Immun 58, 2760-2769.

Andrew, R., Bonnin, J. M. \& Williams, S. (1946). Tick typhus in North Queensland. Med J Aust 2, 253-258.

Babalis, T., Tselentis, Y, Roux, V., Psaroulaki, A. \& Raoult, D. (1994). Isolation and identification of a rickettsial strain related to Rickettsia massiliae in greek ticks. Am J Trop Med Hyg $\mathbf{5 0}$, 365-372.

Bacellar, F., Regnery, R. L., Nuncio, M. S. \& Filipe, A. R. (1995). Genotypic evaluation of rickettsial isolates recovered from various species of ticks in Portugal. Epidemiol Infect 114, $169-178$.

Beati, L. \& Raoult, D. (1993). Rickettsia massiliae sp. nov., a new spotted fever group rickettsia. Int J Syst Bacteriol 43, 839-840.

Beati, L., Roux, V., Ortuno, A., Castella, J., Segura-Porta, F. \& Raoult, D. (1996). Phenotypic and genotypic characterization of spotted fever group rickettsiae isolated from Catalan Rhipicephalus sanguineus ticks. J Clin Microbiol 34, 2688-2694.

Beati, L., Meskini, M., Thiers, B. \& Raoult, D. (1997). Rickettsia aeschlimannii sp. nov.: a new spotted fever group rickettsia associated with Hyalomma marginatum ticks. Int $J$ Syst Bacteriol 47, 548-554.

Bell, E. J. \& Stoenner, H. G. (1960). Immunologic relationships among the spotted fever group of rickettsiae determined by toxin neutralisation tests in mice with convalescent animal serums. J Immunol 84, 171-210.

Bell, E. J., Kohls, G. M., Stoenner, H. G. \& Lackman, D. B. (1963). Non pathogenic rickettsiae related to the spotted fever group isolated from ticks, Dermacentor variabilis and Dermacentor andersoni from Eastern Montana. J Immunol 90, 770-781.

Bella, F., Espero, E., Uriz, S., Serrano, J. A., Allegre, M. A. \& Tort, J. (1991). Randomized trial of 5-day rifampin versus 1-day doxycycline therapy for MSF. J Infect Dis 164, 433-434.

Birtles, R. J. \& Raoult, D. (1996). Comparison of partial citrate synthase gene ( $g l t A$ ) sequences for phylogenetic analysis of Bartonella species. Int J Syst Bacteriol 46, 891-897.
Birtles, R. J., Harrison, T. G., Saunders, N. A. \& Molyneux, D. H. (1995). Proposals to unify the genera Grahamella and Bartonella, with descriptions of Bartonella talpae comb. nov., Bartonella peromysci comb. nov., and three new species, Bartonella grahamii sp. nov., Bartonella taylorii sp. nov., and Bartonella doshiae sp. nov. Int $J$ Syst Bacteriol 45, 1-8.

Black, W. C., IV \& Piesman, J. (1994). Phylogeny of hard- and soft-tick taxa (Acari: Ixodida) based on mitochondrial $16 \mathrm{~S}$ rDNA sequences. Proc Natl Acad Sci USA 91, 10034-10038.

Brenner, D. J., O'Connor, S. P., Winkler, H. H. \& Steigerwalt, A. G. (1993). Proposals to unify the genera Bartonella and Rochalimaea, with descriptions of Bartonella quintana comb. nov., Bartonella vinsonii comb. nov., Bartonella henselae comb. nov., and Bartonella elizabethae comb. nov., and to remove the family Bartonellaceae from the order Rickettsiales. Int J Syst Bacteriol 43, 777-786.

Brown, J. K. M. (1994). Bootstrap hypothesis tests for evolutionary trees and other dendrograms. Proc Natl Acad Sci USA 91, 12293-12297.

Burgdorfer, W., Brinton, L. P., Krinsky, W. L. \& Philip, R. N. (1978). Rickettsia rhipicephali: a new spotted fever group rickettsia from the brown dog tick Rhipicephalus sanguineus. In Rickettsiae and Rickettsial Diseases, pp. 307-316. Edited by J. Kazar, R. A. Ormsbee \& I. N. Tarasevich. Bratislava: Publishing House of the Slovak Academy of Sciences.

Burgdorfer, W., Aeschlimann, A., Peter, O., Hayes, S. F. \& Philip, R. N. (1979). Ixodes ricinus: vector of a hitherto undescribed spotted fever group agent in Switzerland. Acta Trop 39, 357-367.

Chen, D. Q., Campbell, B. C. \& Purcell, A. H. (1996). A new rickettsia from a herbivorous insect, the pea aphid Acyrthosiphon pisum (Harris). Curr Microbiol 33, 123-128.

Crocquet-Valdes, P. A., Weiss, K. \& Walker, D. H. (1994). Sequence analysis of the $190-\mathrm{kDa}$ antigen-encoding gene of Rickettsia conorii (Malish strain). Gene 140, 115-119.

Dessen, P., Fondrat, C., Valencien, C. \& Munier, G. (1990). BISANCE: a French service for access to biomolecular sequences databases. Cabios 6, 355-356.

Drancourt, M., Kelly, P. J., Regnery, R. \& Raoult, D. (1992). Identification of spotted fever group rickettsiae using polymerase chain reaction and restriction-endonuclease length polymorphism analysis. Acta Virol 36, 1-6.

Eremeeva, M. E., Beati, L., Makarova, V. A., Fetisova, N. F., Tarasevich, I. V., Balayeva, N. M. \& Raoult, D. (1994a). Astrakhan fever rickettsiae: antigenic and genotypic analysis of isolates obtained from human and Rhipicephalus pumilio ticks. Am J Trop Med Hyg 51, 697-706.

Eremeeva, M., Yu, X. \& Raoult, D. (1994b). Differentiation among spotted fever group rickettsiae species by analysis of restriction fragment length polymorphism of PCR-amplified DNA. J Clin Microbiol 32, 803-810.

Eremeeva, M., Balayeva, N., Roux, V., Ignatovitch, V., Kotsinjan, M. \& Raoult, D. (1995). Genomic and protein characterization of strain $\mathrm{S}$, a rickettsia isolated from Rhipicephalus sanguineus ticks in Armenia. J Clin Microbiol 33, 2738-44.

Felsenstein, J. (1989). PHYLIP-phylogeny inference package (version 3.2). Cladistics 5, 164-166.

Gilmore, R. D., Jr, Cieplak, W., Jr, Policastro, P. F. \& Hackstadt, T. (1991). The 120 kilodalton outer membrane protein $(\mathrm{OmpB})$ of Rickettsia rickettsii is encoded by an unusually long open reading frame: evidence for protein processing from a large precursor. Mol Microbiol 5, 2361-2370. 
Goldwasser, R. A., Steiman, Y., Klinberg, W., Swartz, T. A. \& Klinberg, M. A. (1974). The isolation of strains of rickettsiae of the spotted fever group in Israel and their differentiation from other members of the group by immunofluorescence methods. Scand J Infect Dis 6, 53-62.

Golinevitch, H. (1960). A propos de la différenciation de quelques rickettsies du groupe de la fièvre pourprée à tiques. Arch Inst Pasteur Tunis 37, 13-22.

Hookey, J. V., Bryden, J. \& Gatehouse, L. (1993). The use of $16 \mathrm{~S}$ rDNA sequence analysis to investigate the phylogeny of Leptospiraceae and related spirochaetes. J Gen Microbiol 139, 2585-2590.

Huebner, R. J. \& Armstrong, C. (1946). Rickettsialpox - a newly recognized rickettsial disease. I. Isolation of the etiological agent. Public Health Rep 61, 1605.

Hughes, A. L. (1991). Circumsporozoïte protein genes of malaria parasites (Plasmodium spp.): evidence for positive selection on immunogenic regions. Genetics 127, 345-363.

Jukes, T. H. \& Cantor, C. R. (1969). Evolution of protein molecules. In Mammalian Protein Metabolism, vol. 3, pp. 21-132. Edited by H. N. Munro. New York: Academic Press.

Kelly, P. J., Beati, L., Matthewman, L. A., Mason, P. R., Dasch, G. A. \& Raoult, D. (1994). A new pathogenic new spotted fever group rickettsia from Africa. J Trop Med Hyg 97, 129-137.

Kimura, M. (1980). A simple method for estimating evolutionary rate of base substitutions through comparative studies of nucleotide sequences. $J$ Mol Evol 16, 111-120.

Kumar, S., Tamura, K. \& Nei, M. (1993). MEGA: molecular evolutionary genetics analysis, version 1.01. Pennsylvania State University, University Park.

Marrero, M. \& Raoult, D. (1989). Centrifugation-shell vial technique for rapid detection of Mediterranean spotted fever rickettsia in blood culture. Am J Trop Med Hyg 40, 197-199.

Martinez-Murcia, A. J., Benlloch, S. \& Collins, M. D. (1992). Phylogenetic interrelationships of members of the genera Aeromonas and Plesiomonas as determined by $16 \mathrm{~S}$ ribosomal DNA sequencing: lack of congruence with results of DNADNA hybridizations. Int $J$ Syst Bacteriol 42, 412-421.

Nei, M. \& Gojobori, T. (1986). Simple methods for estimating the numbers of synonymous and nonsynonymous nucleotide substitutions. Mol Biol Evol 3, 418-426.

Olsen, G. J. \& Woese, C. R. (1993). Ribosomal RNA: a key to phylogeny. $F A S E B J 7,113-123$.

Philip, R. N., Casper, E. A., Burgdorfer, W, Gerloff, R. K., Hugues, L. E. \& Bell, E. J. (1978). Serologic typing of rickettsiae of the spotted fever group by microimmunofluorescence. I Immunol 121, 1961-1968.

Philip, R. N., Casper, E. A., Anacker, R. L., Cory, J., Hayes, S. F., Burgdorfer, W. \& Yunker, C. E. (1983). Rickettsia bellii sp. nov.: tick-borne rickettsia, widely distributed in the United States, that is distinct from the spotted fever and typhus biogroups. Int $J$ Syst Bacteriol 33, 94-106.

Raoult, D. \& Roux, V. (1997). Rickettsioses as paradigms of new or emerging infectious diseases. Clin Microbiol Rev 10, 694-719.

Regnery, R. L., Spruill, C. L. \& Plikaytis, B. D. (1991). Genotypic identification of rickettsiae and estimation of intraspecies sequence divergence for portions of two rickettsial genes. $J$ Bacteriol 173, 1576-1589.

Rehacek, J. (1984). Rickettsia slovaca, the organism and its ecology. Acta Sci Nat Acad Bohemoslov Brno 18, 1-50.

Rikihisa, Y., Kawahara, M., Wen, B., Kociba, G., Fuerst, P.,
Kawamori, F., Suto, C., Shibata, S. \& Futohahi, M. (1997). Western immunoblot analysis of Haemobartonella muris and comparison of $16 \mathrm{~S}$ rRNA sequences of $H$. muris, $H$. felis, and Eperythrozoon suis. J Clin Microbiol 35, 823-829.

Robertson, R. G. \& Wisseman, C. L., Jr (1972). Tick-borne rickettsiae of the spotted fever group in West Pakistan. II. Serological classification of isolates from West Pakistan and Thailand: evidence for two new species. Am J Epidemiol 97, $55-64$.

Roux, V. \& Raoult, D. (1995). Phylogenetic analysis of the genus Rickettsia by $16 \mathrm{~S}$ rDNA sequencing. Res Microbiol 146, 385-396.

Roux, V., Rydkina, E., Eremeeva, M. \& Raoult, D. (1996a). Citrate synthase gene comparison, a new tool for phylogenetic analysis, and its application for the rickettsiae. Int $J$ Syst Bacteriol 47, 252-261.

Roux, V., Fournier, P.-E. \& Raoult, D. (1996b). Identification of the spotted fever group rickettsiae by sequencing and analysis of restriction fragment length polymorphism of PCR amplified DNA of the gene encoding the protein rOmpA. J Clin Microbiol 34, 2058-2065.

Roux, V., Bergoin, M., Lamaze, N. \& Raoult, D. (1997). Reassessment of the taxonomic position of Rickettsiella grylli. Int J Syst Bacteriol 47, 1255-1257.

Saitou, N. \& Nei, M. (1987). The neighbor-joining method: a new method for reconstructing phylogenetic trees. Mol Biol Evol 4, 406-425.

Smith, N. H., Maynard Smith, J. \& Spratt, B. G. (1995). Sequence evolution of the por $\mathrm{B}$ gene of Neisseria gonorrhoeae and Neisseria meningitidis: evidence of positive Darwinian selection. Mol Biol Evol 12, 363-376.

Stothard, D. R. \& Fuerst, P. A. (1995). Evolutionary analysis of the spotted fever and typhus groups of Rickettsia using $16 \mathrm{~S}$ rRNA gene sequences. Syst Appl Microbiol 18, 52-61.

Sumner, J. W., Sims, K. G., Jones, D. C. \& Anderson, B. E. (1995). Protection of guinea-pigs from experimental Rocky Mountain spotted fever by immunization with baculovirus-expressed Rickettsia rickettsii rOmpA protein. Vaccine 13, 29-35.

Tamura, A., Ohashi, N., Urakami, H. \& Miyamura, S. (1995). Classification of Rickettsia tsutsugamushi in a new genus, Orientia gen. nov., as Orientia tsutsugamushi comb. nov. Int J Syst Bacteriol 45, 589-591.

Uchida, T., Uchiyama, T., Kumano, K. \& Walker, D. H. (1992). Rickettsia japonica sp. nov., the etiological agent of spotted fever group rickettsiosis in Japan. Int $J$ Syst Bacteriol 42, 303-305.

Vishwanath, S. (1991). Antigenic relationships among the rickettsiae of the spotted fever and typhus groups. FEMS Microbiol Lett 65, 341-344.

Vishwanath, S., McDonald, G. A. \& Watkins, N. G. (1990). A recombinant Rickettsia conorii vaccine protects guinea pigs from experimental boutonneuse fever and Rocky Mountain spotted fever. Infect Immun 58, 646-653.

Walker, D. H., Liu, Q. H., Yu, X. J., Li, H., Taylor, C. \& Feng, H. M. (1992). Antigenic diversity of Rickettsia conorii. Am J Trop Med Hyg 47, 78-86.

Weisburg, W. G., Dobson, M. E., Samuel, J. E. \& 7 others (1989). Phylogenetic diversity of the rickettsiae. $J$ Bacteriol 171, 4202-4206.

Weisburg, W. G., Barns, S. M., Pelletier, D. A. \& Lane, D. J. (1991). 16S ribosomal DNA amplification for phylogenetic study. $J$ Bacteriol 173, 697-703. 
Weiss, E. \& Moulder, J.W. (1984). Order I. Rickettsiales Gieszczkiewicz 1939, 25AL, p.687-701. In Bergey's Manual of Systematic Bacteriology, vol. 1, pp. 687-703. Edited by N. R. Krieg and J. G. Holt. Baltimore: Williams \& Wilkins.

Werren, J. H., Hurst, G. D., Zhang, W., Breeuwer, J. A. J., Stouthamer, R. \& Majerus, M. E. N. (1994). Rickettsial relative associated with male killing in the Ladybird Beetle (Adalia bipunctata). J Bacteriol 176, 388-394.
Wilson, A. C., Carlson, S. S. \& White, T. J. (1977). Biochemical evolution. Annu Rev Biochem 46, 573-639.

Woese, C. R. (1987). Bacterial evolution. Microbiol Rev 51, 221-271.

Yu, X., Jin, Y., Fan, M., Xu, G., Liu, Q. \& Raoult, D. (1993). Genotypic and antigenic identification of two new strains of spotted fever group rickettsiae isolated from China. J Clin Microbiol 31, 83-88. 